\title{
Power Distribution Network Carrier Communication Route Hybrid Algorithm Based on Ant Colony
}

\author{
Ya Li ${ }^{1, a}$, Rusen Fan ${ }^{2, b}$, Wei Jiang ${ }^{1, c}$, Junjie Yang ${ }^{1, d}$ \\ ${ }^{1}$ School of Shanghai University of Electric Power, Shanghai 200090, China; \\ ${ }^{2}$ State Grid Shanghai Qingpu Power Supply Company, Shanghai 200090, China; \\ adqly2013@126.com, banrusen107@163.com, 'zzm7406@sina.com, diamyjj@163.com
}

Keywords: Electricity information acquisition system, ant colony algorithm, genetic algorithm.

\begin{abstract}
In order to further improve the electricity information acquisition system performance, we put forward a kind of Routing Arithmetic Based on Hybrid Algorithm of Ant Colony Algorithm with Genetic and Simulated Annealing. The algorithm takes the hops, latency and packet loss rate factors into the path overhead cost as the optimal target, and the communication distance between the two nodes and the quality of the communication signal as the constraint conditions to solving the value of the minimum cost routing Generation under these conditions. The algorithm also improved the ant transfer rules and ant colony algorithm pheromone update rule. At the same time, in order to avoid the premature phenomenon of ant colony algorithm, we using genetic algorithm for population initialization, and combined with simulated annealing algorithm to solve the problem of genetic algorithm. The MATLAB simulation show that this algorithm has the advantages of low cost, high reliability and fast convergence to the optimal target and can effectively solve the problem of routing stability, for improving the reliability of power line carrier communication has a great significance.
\end{abstract}

\section{Introduction}

Due to the complex power line structure of distribution network, the power line carrier communication inevitably affected by the influence such as channel frequency selectivity, low impedance, strong noise interference, weak signal ${ }^{[1]}$ and other factors. All of these will cause some terminal node to add or withdraw with without rules, resulting to PLC network logic topology changes. In addition, the quality of the carrier communication signal is influenced by the channel, which seriously restricts the real-time performance and reliability of the carrier communication. Therefore, it is needed to establish a fast and efficient distribution network carrier communication routing based on network topology.

In the physical layer, reference [2] has a black box device which has the capability of communication evaluation, but the performance is unstable and easily affected by the presence of the environmental impact; reference [3] selected cluster head based on the lowest ID clustering algorithm, although the algorithm can quickly got a shortest path, but this path information is not reliable, and the path is single, the survivability is poor.

This paper starts from the network layer to improve the adaptability and reliability of the network. The paper takes the minimum path overhead as the optimal target, and takes the communication distance between the two nodes and the quality of the communication signal as the constraint conditions, on the basis of this we put forward a kind of routing arithmetic based on a hybrid algorithm of ant colony algorithm, genetic and simulated annealing. The algorithm takes the hops, latency and packet loss rate factors as the path overhead cost, in order to prevent the occurrence of local target optimization and other target is poor, in the ant colony algorithm using distance and signal quality as the heuristic factor, which not only consider the distance constraint but also increases the signal quality constraints; furthermore, we introduced the genetic algorithm to update the pheromone of ant colony continuously, and eliminate the worst path in the iterative process gradually. Considering the robustness of the ant colony algorithm, the genetic algorithm and the simulated annealing algorithm are introduced to initialize the population, which can improve the adaptability of the ant colony algorithm. Therefore, the influence of the network transmission medium on the 
communication channel will change the network topology, the hybrid algorithm of this paper have the advantages of high reliability, strong self-healing and fast convergence for the optimal objective.

\section{Logic Topology Structure of Distribution Network}

The typical electricity information acquisition system structure is mainly divided into three levels: concentrator, collector and meters. Each concentrator is connected with the transformer low-voltage three-phase outlet, and most of the PLC system is composed by many collectors with distributed structure $^{[4]}$ controlled by one concentrator; in addition, the concentrator as the source routing node and the collector has the relay function, which they communicate with the power line carrier; collector and the meter communicate with the RS485, all of electrical information sent to the remote control center through the GPRS after concentrator summary all courts electricity information.

The logical topology structure of distribution network has closely relation to its application places $^{[5]}$, the low voltage distribution network using three-phase power but each user most of the use of single-phase power supply, the three phases are tied and independent of each other in the logical topology, so it can be studied in the actual research, In the practical application, the logic topology structure of distribution network most use the tree topology structure as shown in Fig 1, among the node 2-50 on behalf of the collector.

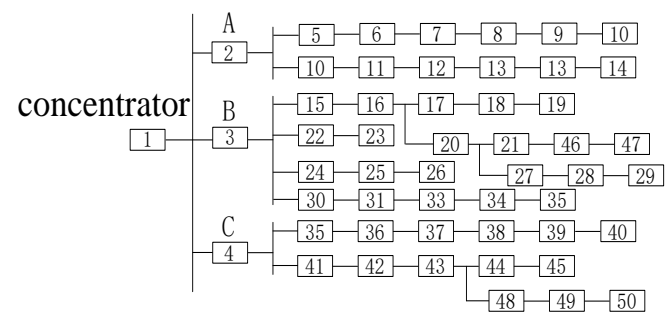

Fig. 1 Distribution network logic structure diagram

\section{Hybrid Routing Algorithm}

The Improvement of Ant Colony Algorithm. Because of the convergence rate of ant colony algorithm is greatly influenced by the initialization pheromone concentration. If the pheromone concentration is low, the ant colony algorithm is easy to premature and convergence to a local optimal solution; if the initial pheromone concentration is higher, the pheromone concentration on the search direction guidance performance lower. The ant colony algorithm to search can in a wide range but convergence speed is slow. The genetic algorithm has a faster search speed, but the search precision is limited and is easy to appear the problem of internal closed loop in the process of searching inside, the simulated annealing algorithm can effectively solve the problem of the internal loop of genetic algorithm, so that, the hybrid algorithm can overcome its own shortcomings and then apply to the routing network. Therefore, we can first to search the sub optimal solution based on the genetic algorithm and the simulated annealing algorithm, and based on this to initialized the pheromone concentration, and to speed up the convergence rate of the ant colony algorithm significantly.

The Initial Population. The process of combined ant colony algorithm, genetic algorithm with simulated annealing algorithm is the process of initial population, after setting up the path gather, we randomly select a path to crossover, mutation, simulated annealing, and constantly update the ant colony transfer rules and local or global pheromone, to prevent the ant colony from falling into local optimum. The specific steps for the initialization of the population are as follows:

First, in a given group $P(k)$, set the initial temperature $t_{0}$; in group $P(k)$, the probability of each chromosome is accepted by simulated annealing from the formula(1):

$$
A_{i j}\left(t_{k}\right)=\min \left\{1, \exp \left(-\frac{f(j)-f(i)}{t_{k}}\right)\right\}
$$


Where $t_{k}=t_{0}, f_{i}, f_{j}$ is the state value in the $i$ and $j$ moments of the group, after the process of continuous iteration selected the new group new $P(k+1)$ according to the size of the probability; then calculate the fitness function in the new group. The formula is as shown in(2):

$$
f_{i}\left(t_{k}\right)=\exp \left(-\frac{f(j)-f_{\min }}{t_{k}}\right)
$$

The higher fitness chromosome is selected to compose the final needs of the population, and then mating, mutation and selection, to initialize the population.

The Selection of Ant Colony Routing. The ant of $k$ in the node of $i$ selected the node of $j$ as the next hop probability with the formula $P_{i j}{ }^{k}(t)$ in the PLC, the state transition rule ${ }^{[6]}$, as shown in the formula (3):

$$
j=\left\{\begin{array}{l}
\arg \max \left[\left[\tau_{i j}(t)\right]^{\alpha}\left[\eta_{i j}(t)\right]^{\beta}\right], q \leq q_{0} \\
s, q>q_{0}
\end{array}\right.
$$

Where in the formula(3), $q \in[0,1]$ is a random number, $q_{0} \in[0,1]$ is a fixed value. Its value determines the probability that the next hop has passed the path or the path has not been passed. It can effectively expand the range of the algorithm and avoid the premature; $\tau_{i j}(t)$ is the pheromone function value at the moment $t, \eta_{i j}(t)$ is the heuristic information function value at the moment $t$, the calculation as shown in the formula(4); $\alpha$ is the influence factor of pheromone concentration, which indicates the influence of pheromone concentration on the next hop selection probability, $\beta$ is the heuristic information factor, we usually uses roulette strategy to get $s$, which is probability of $P_{i j}{ }^{k}(t)$ being selected of the individual $k, P_{i j}{ }^{k}(t)$ can be expressed as a percentage ${ }^{[7]}$ of the sector in the disk area occupied just as the in the formula(5):

$$
\begin{aligned}
& \eta_{i j}(t)=A * \operatorname{sig}_{i j}+B * d i s_{i j} \\
& P_{i j}^{k}(t)=\left\{\begin{array}{l}
\frac{\tau_{i j}^{\alpha}(t) \cdot \eta_{i j}^{\beta}(t)}{\sum_{s \in A_{\mathrm{k}}} \tau_{i j}^{\alpha}(t) \cdot \eta_{i j}^{\beta}(t)}, j \in A_{\mathrm{k}} \\
0, j \notin A_{\mathrm{k}}
\end{array}\right.
\end{aligned}
$$

Where in the formula(4), $s i g_{i j}$ is the signal quality in communication between node $i$ and $j$, which can be get by extracting signal quality flag byte, and it is range of $0-15$, the greater the number of signal quality, the better the quality of the channel and the better the communication reliability is, such as the number of 0 indicates the communication failure, 1 indicates that the communication is successful but the signal quality is the weakest, the 15 indicates that the communication is successful and the signal quality is the best, in order to ensure the reliability of communication, when the signal quality of two node communication is not less than 8 , it is considered as an effective and reliable communication; in contrast, signal quality is less than 8 and cannot be communication. dis ${ }_{i j}$ is the distance between two points, $A$ and $B$ represent the weight factor, according to the actual installation location of the site. In the ant colony algorithm considering the actual situation of signal quality and distance can effectively avoid long-distance transmission signal attenuation effect on reliable communication, introducing the signal quality can comprehensive two nodes optimal path for transmission, so as to achieve the best channel condition and the high communication quality requirements. $A_{k}$ is represent the gather of node $i$ and the neighbor node $j$ as shown in the formula(5).

The Selection of Objective Function. The algorithm using a combination of hops, delay and packet loss rate as the objective function of path cost, taking the communication distance and extraction signal quality of the node after the successful communication as the constraint conditions and solving the optimal path allocation. The optimal target as shown in the formula (6):

$$
\left\{\begin{array}{l}
L=\min \left(\lambda_{1} \sum_{i=1}^{d} e_{i j}(p)+\lambda_{2} \cos (p)\right) \\
p=\left(p_{1}, p_{2}, p_{3} \cdots p_{N}\right)
\end{array}\right.
$$

Where in the formula(6), $L$ represents the path overhead, the function value is the weighted average of the number of hops, delay, and packet loss. The number of any path $P=\left(P_{1}, P_{2}, P_{3} \cdots P_{N}\right)$ 
of the starting node to the target node hops as shown in the formula (7); $\cos (p)$ is the weighted sum of time delay and packet loss rate as shown in the formula (8). In the objective function, the $\lambda_{1}$ and the $\lambda_{2}$ are the weight coefficients, and meet the requirement of $\lambda_{1}+\lambda_{2}=1$.

$$
\begin{aligned}
& T=\sum_{i=1}^{d} e_{i j}(p) \\
& \cos p_{i}=\mu_{d} w_{i}^{d}+\mu_{l} w_{i}^{l}
\end{aligned}
$$

Where in the formula(8), $w_{i}{ }^{d}$ is the weighted coefficient of time delay, $w_{i}^{l}$ is the weighted coefficient of packet loss ratio, the time delay of $u_{d}$ as show in the formula (9); $u_{l}$ is the packet loss as show in the formula (10);

$$
\begin{aligned}
& u_{d}(s, d)=\sum \operatorname{delay}\left(N_{i}, N_{j}\right)+\sum \operatorname{delay}\left(N_{n}\right) \\
& u_{l}(s, d)=1-\prod\left[1-\operatorname{Packetloss}\left(N_{i}, N_{j}\right)\right]
\end{aligned}
$$

Where in the formula(9), $\sum \operatorname{delay}\left(N_{i}, N_{j}\right)$ is the tansmission delay between node $i$ and $j ; \sum \operatorname{delay}\left(N_{n}\right)$ indicates processing delay for a node to respond to another node $n$. Where in the formula (10): Packetloss $\left(N_{i}, N_{j}\right)$ is the packet loss between node $i$ and $j$.

The Pheromone Concentration Updating. In this paper, the pheromone update method of ant colony algorithm is improved, and the optimal and worst path is introduced. We selecting the optimal path and avoiding the worst path. The pheromone update method as shown in the formula (11):

$$
\tau(r, s)=(1-\rho(\tau(r, s)) * \tau(r, s))+\Delta c
$$

Where in the formula(11), $\rho \in[0,1], \rho(\tau(r, s))$ is the factor of local pheromone evaporation. $\Delta c$ is the pheromone concentration increment, and the updating only when the path is the best or worst path, the other condition is 0 , it is as shown in the formula (11):

Respectively, when the $(r, s)$ is a local or global optimal path:

$$
\left\{\begin{array}{l}
\Delta c=\rho * \Delta \tau(r, s) \\
\Delta \tau(r, s)=Q / L
\end{array}\right.
$$

when the $(r, s)$ is a local or global worst path:

$$
\left\{\begin{array}{l}
\Delta c=-[\rho * \Delta \tau(r, s)] * \Delta \tau(r, s) \\
\Delta \tau(r, s)=Q / L
\end{array}\right.
$$

Where in the formula(12), $Q$ is the sum of pheromone concentrations on the path. After the ants choose the next hop for local pheromone update, you can make more ants constantly release pheromone, and update the path information in a timely manner, to avoid algorithm falling into local optimum. We introduced the optimal and worst path in the local and global pheromone update, and the objective function is introduced into the optimal path. This process is iterated, so that the ants can find the optimal path.

\section{The Algorithm Simulation and Verification}

In this paper, the feasibility of the algorithm is verified by MATLAB simulation. The topology structure of the analog electric network is 50 points in the range of $100 * 100$ MATLAB. And the number is in turn, in which the node 1 is the carrier primary node or the concentrator, 2-50 is the carrier from the collector node or meter, and then construct the adjacency matrix, finally draw the simulated network topology. At last, the initialization parameters of the synthesis algorithm are shown in table 1.

The initial value of each algorithm can be obtained according to the parameters of table 1 , and the simulation results are shown in Fig 2. When only used the distance as the ant colony algorithm's heuristic factor, the packet loss rate is shown as a curve $f_{1}$, on the contrary, considering the distance and signal quality of ant colony algorithm, the packet loss rate as shown in curve $f_{2}$, compared with $f_{1}$ and $f_{2}$, the contrast curve shows that the channel parameters can be adjusted after the signal quality is increased, and the data packet reception rate is obviously improved. In this paper, based on the hybrid routing algorithm, we use the distance and signal quality as the heuristic factor to improve the ant 
Table 1 The hybrid algorithm initialization parameter

\begin{tabular}{ccc}
\hline Parameter & Parameter Value & Parameter Meaning \\
\hline$m$ & 50 & The number of ants \\
$\alpha$ & 1 & Pheromone factor \\
$\beta$ & 2 & Heuristic information factor \\
$N_{\max }$ & 100 & The maximum number of iterations of ant colony algorithm \\
$\rho$ & 0.7 & Global / local pheromone evaporation rate \\
$t_{0}$ & 10 & The initial temperature value \\
$d$ & 2 & Transmission delay weighting factor \\
$l$ & 3 & Packet loss rate weighting factor \\
$A$ & 0.4 & Signal quality weighting factor \\
$B$ & 0.6 & Distance weight factor \\
\hline
\end{tabular}

colony algorithm's transfer rule. By introducing the best and worst path improved ant colony algorithm's information pheromone updating rule and the combination of genetic algorithm and simulated annealing algorithm of ant colony are used to initialize the population; then we use crossover, mutation and simulated annealing, and other operations, now we can see the network packet loss rate is the lowest, as shown in the curve $f_{3}$.

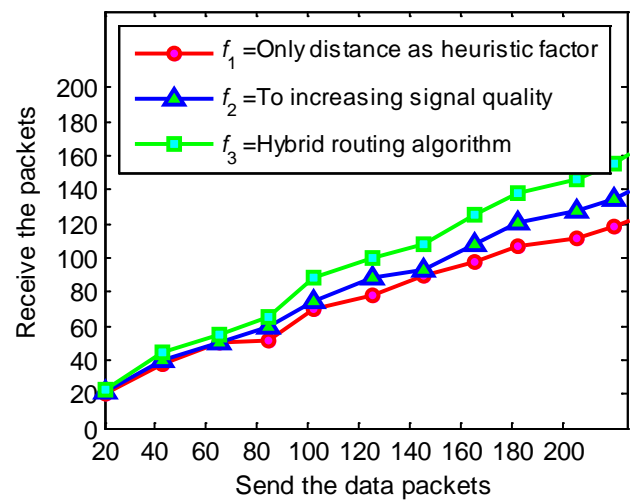

Fig. 2 The network throughput

The network transmission delay as shown in Fig 3, under in consideration of signal quality and global, local mixing conditions optimal routing paths the channel quality has improved significantly, and reduced the cumulative amount of delay. The combination of genetic algorithm and simulated annealing algorithm can effectively improve the adaptability of ant colony algorithm, and can select the population with the highest degree of fitness, which can improve the reliability and reduce the ant's stagnation phenomenon in the process of searching path, as shown in curve $f_{1}$. However, in the single target routing algorithm, the algorithm has not considered the packet loss rate, channel condition and so on, and not combined with genetic algorithm or simulated annealing algorithm, in this case, we get the curve as shown in curve $f_{2}$; to compared with these curves, this paper shows that the hybrid routing algorithm reduces the time transmission delay significantly.

The convergence of the algorithm is as shown in Fig 4. In this paper, the convergence curve of the improved multi-objective hybrid routing algorithm is as shown in curve $f_{1}$. The single target routing algorithm based on ant colony algorithm only takes into account the convergence curve of the hop count, as shown in $f_{2}$. From the Fig 4, we can see that the improved multi-objective hybrid routing algorithm can quickly find the optimal path and save the network overhead, and which can effectively improve the communication reliability and speed of the network routing node.

\section{Summary}

In this paper, in order to improve the reliability of routing and communication of electricity information acquisition system, the multiple objective functions set the minimum number of hops, time delay and packet loss rate as the path overhead, added the constraints of distance and signal quality. By using the signal quality to improve the transfer rule of ant colony algorithm, then, the introduction of the best and worst path to improve and update principle to improve its reliability. The 


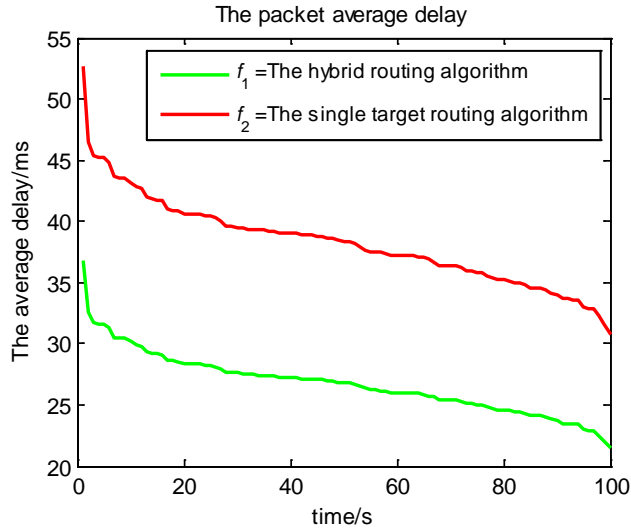

Fig. 3 The comparison of propagation delay

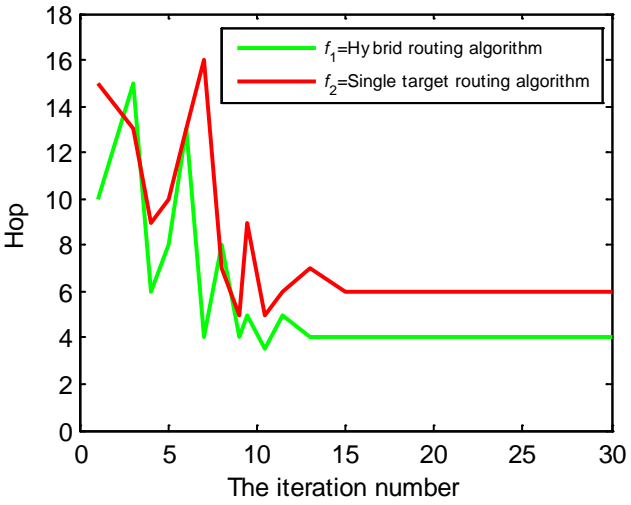

Fig. 4 The convergence curve

Heuristic genetic algorithm's crossover and mutation improved the ant colony algorithm's prematurity and premature local optimum shortcomings, then, combined with the simulated annealing algorithm to solve the problem of the genetic algorithm's inside the closed loop, and to speed up the late evolutionary search capability. The MATLAB simulation results show that this algorithm has the advantages of fast convergence, strong reliability, low cost and so on. It can meet the requirement of automatic routing.

\section{Acknowledgements}

Support for this program is provided by the National Natural Science Foundation of China (61202369 and 61401269), Shanghai Natural Science Foundation (14ZR1417400), Shanghai Technology Innovation Project (13160500900) and Innovation Program of Shanghai Municipal Education Commission(13YZ102). Corresponding author: Wei Jiang zzm7406@sina.com.

\section{References}

[1] Cheng Xiaojuan, Geng Xueying Study on Dynamic Routing Algorithm for Low Voltage Power Line Carrier Communication, Heilongjiang Electric Power. 2013,35(1):6-8,15.

[2] Sun Hongliang, Hou Xingzhe, Ye Jun, Development of Low-Voltage Power Line Carrier Route Testing System, Proceedings of the Electrical Measurement \& Instrumentation. 2013,50(571):101-103.

[3] Qi Jia-jin, Liu Xiaosheng, Xu Dianguo, Cluster-based Routing and Reconstruction Method of Power Line Communication over Lower-voltage Distribution, Proceedings of the CSEE. 2008,28 (4) :65-71.

[4] Wang Ning, Yu Chuanwei Gu, Ying Design of Meter Reading System Based on Power Line Carrier, Proceedings of the Electronics Quality. 2015,04:25-28.

[5] Zhu Qigui, Yzng Ye, Mou Xianmin, Monitoring System of 10k V Distribution Network by Power Line Carrier, Proceedings of the Electrical Measurement \& Instrumentation. 2014, 51(2):10-13.

[6] Zhao Kaixin, Wei Yong, Wang Dongshu, Application of Improved antColony Algorithm in DSR Routing Protocol, Proceedings of the Fire Control \& Command Control. 2015, 40(7):135-139.

[7] Li Tian, Shi Xin, Li Yongqian, Research on routing optimization technique of wireless sensor networks based on power lines monitoring, Proceedings of the Electrical Measurement \& Instrumentation. 2015, 52(21):6-10. 